\title{
Survey on Different Methods of Digital Audio Watermarking
}

\author{
Prof. Amruta M. Chore*, Prof. Neha Tiwari** \\ *(Department of Electronics and Telecommunication, Pune University, Pune \\ **(Department of Computer Engineering, Pune University, Pune
}

\begin{abstract}
The significant progress of the technology gives the full access to the digital data for retransmitting and reproduction with comfort. Since the benefits of such progress is easily available, they equally immune to some illegal manipulation of data. So there is necessity arises for the protection of digital data from unauthorized users. The digital audio watermarking technique is new technology among different watermarking techniques which provides successful solutions to problems occurred from some digital attacks. Basically watermarking is the scheme in which binary information is embedded into the original signal. The major concern of the audio watermarking scheme is to provide the proof of ownership to the owner and to provide protection for embedded data. This paper provides concise analysis of different existing audio water.
\end{abstract}

Keywords: Audio Watermarking, Digital Data, Ownership, , Signal ,Unauthorized.

\section{INTRODUCTION}

In this new era with the growth of the communication networks and devices like the CD, pen drive, hard disc and the online storage options like cloud it provides to get access for digital data. With the development of the technology, music industry is going for the research in the field of storage space, recovery and distribution model for songs to get successful access for the broadband transmission as well as for digitization of the audio signals. The transmission of digital audio is easy due to which it provides unauthorized access to digital technology particularly in case of illegal copy, downloads and allocation of multimedia work. That is why the importance of certification, content verification, authorized copying and content security in digital audio has become more perceptible and sensitive issue. Digital watermarking technique gives the better and robust solution for ownership problem. It is important to keep the official document of the digital data which is in the form of intellectual properties Digital watermarking technique embed copyright information or watermark into the original audio signal. The implanted information should be undetectable and secure. These embedded watermarks are not easy to eliminate just by changing or damaging the original audio signal. While digital audio watermarking is a scheme in which watermark is to be embedded into the digital audio signal and embed should not alter the value of the original digital audio signal. It provides the significant similarity for modified signal with the original signal. Digital audio watermarking technique is new as compare to other watermarking technique such as image watermarking, video watermarking. Human auditory system plays an important role in case of audio watermarking because it has wider dynamic range as compared to human visual system. Although it has huge dynamic range, HAS contains a little differential range, i.e. High range sounds generally masked weaker sounds. Additionally, HAS is not sensitive to a stable relative phase shift in a fixed audio signal and some spectral distortions interpret as usual, non-annoying ones.

Most of the recent audio watermarking can be divided in two parts:

1. Time domain watermarking.

2. Transform domain watermarking.

\section{TIME DOMAIN WATERMARKING}

It is first watermarking technique which was find out by researchers. Insert in the temporal domain means that the watermark is implanted directly into the host audio by changing the array or mixing PN pattern to the audio data. This technique contains Low bit coding such as LSB coding. It is achieved by the camouflage of noises intrinsic to digital signal acquisition process. It require changes in the LSB of every examined of the digitized audio stream. As this technique is simple to design and can insert large data, however it is susceptible to even a slight audio distortion, such as simple file conversion. It also cause audio degradation because of rapid changes especially in LSB.

\section{TRANSFORM DOMAIN AUDIO WATERMARKING:}

Audio watermarking techniques which work in transform domain, get the benefit of audio masking features of Human Auditory System (HAS) to install an inaudible watermark signal in digital audio signals. Transform audio signal from time domain 
enables watermarking system to insert the watermark into perceptually important components. This gives the system with a high level of toughness and in audibleness.

\subsection{Human Auditory System}

Numerous works has been done on digital watermarking of different media such as image and video, but this particular work will centre on digital watermarking of audio file. Inaudible watermarks are made possible by exploiting explanation of the Human Auditory System (HAS). Audiowatermarking is especially not easy as compare to image watermarking because the HAS is distant more sensitive than the visual system. Human ear is more sensitive than other sensory organs. If a signal is kept in below fig. 1 the threshold of sensitivity, then the watermark will be inaudible. Above $2 \mathrm{kHz}$, the HAS focus more on the temporal envelope of an audio signal than the actual structure. Thus, small changes in the band above $2 \mathrm{kHz}$ are less likely to be noticed by a human listener.

\subsection{Different objectives followed by watermarking scheme}

Perceptual transparency: There are two types of method available for embedding watermark in original audio file. This two methods are either be perceptible or imperceptible. Imperceptible watermarks are preferred in audio files because it gives better robustness. Robustness: Robustness define the defence capability of the original signal in which the watermark is embedded for the proof of ownership, it should be able to handle all sorts of attacks such as signal processing attacks, noise like Gaussian noise etc.

Capacity: The data stored in the original signal must be large enough to provide enough space in the signal for embedding watermark in an effective manner.

Reliability: Is determined by the robustness and detection rate of the watermark. A watermark has to be robust against intentional and unintentional attacks .The detection rate of watermark should be used to determine whether the original signal is altered by some external attacks or not. The attacks on the audio files include D to A, A to D conversions, noise addition, BPF, time-scale modification, addition of echo and sample rate conversion. If the quality of the watermarked signal after the attacks is not significantly distorted, then it proves the reliability of the watermark signal.

Security: Secret keys are essential for security issues and are included in many watermarking systems to protect the watermark information from undesirable alterations or even removal. There are two kinds of secret keys: unrestricted-keys and restricted keys. Unrestricted-keys are those in which the same key is known and used in different watermarks, whereas restricted-keys are used only in a specific watermark. Speed: Watermarking may be used in audio streaming. The watermark inserting and extracting processes must be quick enough to suit these applications.

\subsection{Performance Evaluation Measures}

For the evaluation of the performance of different watermarking scheme, various parameters can be used. There can be subjective tests which can be used to check the imperceptibility, where the listeners are made to listen the watermarked audio file in order to identify the similarities and dissimilarities between the original and the watermarked audio file. This is done using ITU-R grade, which is a 5-grade impairment scale in which the Mean Opinion Score (MOS) is calculated. Different grades scale, also can be used for evaluating the imperceptibility of the watermarked audio. Objective tests are also performed to evaluate the performance. Bit Error Rate (BER) is used to evaluate the robustness of the algorithm. Greater the BER lesser will be the robustness. To measure the perceptual quality of the audio signal, Signal to Noise Ratio (SNR) and Peak Signal to Noise Ratio (PSNR) can also be calculated. These parameters are used to amount of data that has been corrupted because of the noise. Error is the difference between calculated value and the actual value. To measure the error in the watermarked audio file, Mean squared Error (MSE) is used. It is the average of square of 'errors'. Certain similarity tests are also performed to evaluate the amount of similarity present between the watermark extracted and the actual watermark used. Normalized Cross Correlation (NCC) is used to measure the same. Sim function has the similar role as NCC.

\section{RELATED WORK}

[1] In 2010 Liu Ping Feng, Liang Bin Zheng, Peng Cao proposed a paper which gives the work related to DWT-DCT based blind watermarking algorithm for copyright protection. In this the combination of two algorithm is used, the watermark is first scrambled by Arnold and then the watermark is embedded in a spread spectrum pattern using pseudo random. In this watermark process begins by applying DWT to the original content and then the DCT is applied on subband formed by the DWT, as it is blind watermarking technique host signal is not present at the time of extraction. According to the results this algorithm provides better performance as compared to DCT algorithm in accordance to security and robustness.

[2] In September 2012 HimeurYassine, Boudrabachir and Khelefaziz proposed a paper which gives the work related to hybrid transform audio watermarking 
algorithm .Transform domain techniques provides better performance than time domain because transform domain technique is largely exploited to Human Auditory System In this two powerful transforms are applied on the original audio signal that is Discrete Wavelet transform and Discrete sine transform using double insertion of the watermark. The watermark used in this scheme is gray scale logo image instead of randomly generated Gaussian noise type watermark. Many subjective and objective test have been performed on the proposed watermarking scheme which determines the high quality audio signal quality and robust to different attacks. The advantage of this method is the property of energy compaction of Discrete Sine transform. The Finite Ridgelet Transform also known as FRIT is used for encrypting system to secure the watermarking system.

[3] In 2001 ParaskeviBassia, Ioannis Pitas and Nikos Nikolaidis proposed a paper which gives the work related to audio watermarking algorithm for copyright protection of the audio signal by time domain processing. The watermarking algorithm used in this paper does not require the original signal for the detection process. The audio watermarking algorithm approached in this paper the set of the original digital audio samples is divided in embedding segments in order to further increase the robustness of the detection scheme to various signal domain of the original audio. The watermark signal is created using a key i.e. single number known only to the copyright owner. The watermark embedding depends on the amplitude and frequency of audio signal in a way that reduces the audibility of the watermark signal. The advantage of this method is to provide robust to different to common signal properties like cropping, time shifting, filtering, resampling and re-quantization. The disadvantage of this method is that it is not robust against more sophisticated attack like changing in time scale of the original signal.

[4] In 2012 Teruya Minamoto, Yuji Ogata and Masahiro Sawai proposed a paper which gives the work related to the blind audio watermarking algorithm for embedding watermark in a binary logo form in an audio signal this technique is known as Dyadic Wavelet Transform. Basically one dimensional DYWT method but here in this work two dimensional DYWT method used by constructing an image from an audio signal, the original signal is divided into some parts and arranged these parts in some rows based on the visualization method. The disadvantage of this method is that it is shift variant that is why a watermarking method based on DYWT is expected to be robust against geometrical distortions such as clipping and desynchronization. The advantage of this method is that it gives redundant representation and can be implemented with a fast algorithm, the amount of information that the watermark must carry is higher than in DWT-based methods

[5] In 2015, Guang Hua, Jonathan Goh, and Vrizlynn. L. L. Thing proposed a novel time spread echo-based audio watermarking scheme with optimized imperceptibility and robustness. This work improve imperceptibility by suppressing the frequency response of the kernel in perceptual significant region. This is achieved by reducing low frequency patterns in the PN sequence. However, such suppression is inexact. In contract, we propose to design the echo kernel from finite-impulseresponse (FIR) filter design perspective, where convex optimization is used to obtain a set of filter coefficients to replace the PN or MPN sequence.

[6] In 2014, Arshdeep Kaur, Malay Kishore Datta,K.M.Soni and Nidhi Taneja proposed a blind audio watermarking technique which does not require original signal at the time of detection process. The proposed algorithm is built in wavelet domain using QR decomposition for inserting a watermark in original audio signal. The above method is carried out in frequency domain having advantages such as high payload capacity and robust against different synchronization attack like compression etc. The major disadvantage of time domain technique is that it is immune to different signal processing attacks. The throughput of this method is that it provides high payload capacity for embedding the watermark in an original signal.

[7] In 2014, HoseinFamiTafreshi and Ali AsgharSoltaniFarani proposed a paper on audio watermarking which takes the use of spread spectrum watermarking which is basically modulating method for adding a watermark in a host signal. In this paper spread spectrum technique is used for cyclic shift in the pseudo noise sequence because it gives the advantage in embedding information capacity in the host signal while in traditional spread spectrum technique only one bit information is embedded in each frame. Another advantage of this method is that it maintains the transparency in the watermarked audio signal in silent frames i.e. the frame which has low energy content. Different preprocessing schemes like high pass filtering, linear predictive coding and whitening filter insure that this approach can be utilized for transferring watermark signal through telephone lines. According to the experiment performed on different audio samples it is observed that the traditional spread spectrum technique is more robust against different attacks but when band pass filtering and all pass filtering is taken into consideration then spread spectrum technique is best. [8] In 2013, Jung-HeeSeo and Hung-Bog Park proposed an audio watermarking scheme with the hardware implementation. In the proposed system it gives a real time performance by proper integration 
of the software based watermarking schemes and the available electronic devices. The basic objective of this scheme is to achieve low power performance, real time analysis, stability and imperceptibility. In the hardware based watermarking scheme it uses blind watermarking algorithm to decrease the memory requirements. In this method it embeds watermark in coefficients bits of the low frequency band and as it is the blind approach it extracts that watermark without the original signal. It is implemented in a blackfin processor using embedded module based Labview which is high level graphical language to design the watermark algorithm for embedding in the hardware. But hardware implementation can increase the restriction in case capacity of embedded data and search access time.

\section{V.CONCLUSION}

Audio watermarking is new domain in the area of watermarking. It is much more bulky as compared to the image watermarking. This is because of the fact that the Human ears are way more sensitive as compared to the Human eyes. The level of watermarking increases robustness of the secret information. The audio watermarking is new and has largr scope for research.. In this context Wavelet algorithms provide decent performance. This survey concentrates on developing an audio watermarking technique to detect convergence and robustness, improving watermark imperceptiveness.

\section{REFERENCES}

[1]. Liu Ping Feng, Liang Bin Zheng, Peng Cao, "A DWT-DCT Based Blind Watermarking Algorithm for Copyright Protection", Computer Science and Information Technology (ICCSIT), 2010 3rd IEEE International Conference, vol 7 ,pp 455458,2010 .

[2]. HimeurYassine, BoudraaBachir and KhelalefAziz , "A Secure and High Robust Audio Watermarking Systemfor Copyright Protection," International Journal of Computer Applications (0975 - 8887)Vol 53, No.17,pp 33-39,September 2012.

[3]. ParaskeviBassia, Ioannis Pitas and Nikos Nikolaidis, "Robust Audio Watermarking in the Time Domain," IEEE TRANSACTIONS ON MULTIMEDIA, VOL. 3, NO. 2, pp 232-241, June 2001.

[4]. Teruya Minamoto, Yuji Ogata and Masahiro Sawai , "Visualization of Digital AudioWatermarking based on the Dyadic Wavelet Transform", 2012 Ninth International Conference on Information Technology- New Generations, pp 640-645, 2012.
[5]. Guang Hua, "Time-Spread Echo-Based Audio Watermarking With Optimized Imperceptibility and Robustness", IEEE/ACM Transactions on Audio, Speech, and Language Processing, vol-23,pp 227239,2015.

[6]. ArashdeepKaur,Malay Kishore Dutta,K.M.Soni and Nidhi Taneja, "A High Payload Audio Watermarking Algorithm Robust against Mp3 Compression", Contemporary Computing (IC3), 2014 Seventh International Conference,pp 531$535,2014$.

[7]. HoseinFamiTafreshi, Ali AsgharSoltaniFarani, "Spread spectrum audio watermarking: Enhancement of detection and watermarking capacity", 22nd Iranian Conference on Electrical Engineering (ICEE),pp1477-1482,2014.

[8]. Jung-HeeSeo, Hung-Bog Park, "Hardware Based Real Time Audio Watermarking Using Embedded Module", Journal of Next Generation Information Technology(JNIT) Volume4, Number5, July 2013, doi:10.4156/jnit. vol4. issue5.1 